Ortwin Renn

\title{
Vorsorge: Ein Prinzip zur vorbeugenden Risikoreduktion am Beispiel COVID-19
}

\begin{abstract}
Prevention: The Concept of Preventive Risk Reduction, Illustrated by the COVID-19 Pandemic. Risk assessment and risk management based on the precautionary principle are instruments to protect humans and the environment against uncertain threats and risks. At the same time, however, trade-offs must always be made in order to maintain cultural, economic and social objectives and to honor the principle of proportionality. Notwithstanding the need for continuous risk reduction, society must distance itself from the idea that science can predict all dangerous events and developments and exclude them through preventive action. At best, risk analyses are able to identify the possible threats and uncover weaknesses in the system. In addition, risk assessments help us to make better decisions in the conflict of objectives between the secondary consequences of taking risks (such as exposure in the event of a pandemic) and the secondary consequences of the protective measures taken (economic, social, cultural consequences). In this sense, decisions about acceptability of risks or risk reduction measures always reflect a combination of scientific knowledge with balancing judgments.
\end{abstract}

\section{Einleitung}

Konventionelle Formen der Risikobewertung beruhen auf der Annahme, dass die Wirkungen der zu beurteilenden Handlungen bekannt sind, und zwar im Hinblick auf die maximale Schadenshöhe wie auch im Hinblick auf die Wahrscheinlichkeitsverteilung potenzieller Schäden. Die Schwere und Häufigkeit der Konsequenzen einer Handlung oder eines Ereignisses werden dabei als Maßstab zur Beurteilung ihrer Akzeptabilität herangezogen.

Wie aber soll man Situationen einschätzen, bei denen die Wirkung selbst noch unsicher ist? Wie kann man Gefahren beurteilen, bei denen man die Wahrscheinlichkeit ihres Eintretens nicht oder noch nicht berechnen kann? Wie sollten Risikomanager auf Risiken reagieren, bei denen Schadenswirkungen vermutet werden, gleichzeitig aber die Datenlage fehlt, um die Höhe des Schadens zuverlässig abschätzen zu können? In diesen Situationen kommt das Prinzip der

Ortwin Renn, Institut für Transformative Nachhaltigkeitsforschung (IASS), Potsdam

Ә OpenAccess. (C) 2021 Ortwin Renn, publiziert von De Gruyter. (cc) BY-NC-SA Dieses Werk ist lizenziert unter einer Creative Commons Namensnennung - Nicht kommerziell - Weitergabe unter gleichen Bedingungen 4.0 International Lizenz. https://doi.org/10.1515/9783110713336-026 
Vorsorge zum Tragen (Elliott \& Charnley 2002). Das Prinzip der Vorsorge ist dann angesagt, wenn hohe Ungewissheit bei der Risikoabschätzung vorliegt. Das Konzept der Vorsorge ist also auf das Problem der Ungewissheit bei der Risikoabschätzung bezogen (Bennett 2000). Wie soll man Risiken regulieren, bei denen man über die möglichen Folgen einer zur Debatte stehenden Handlung nichts oder wenig weiß bzw. eine Ungewissheit über die Wahrscheinlichkeitsverteilung besteht?

Diese Situation war zum Beispiel zu Beginn der Coronakrise gegeben. Es herrschte große Unsicherheit darüber, welches Ansteckungsrisiko mit dem neuen Virus verbunden war und wie hoch die Letalität, also die Sterblichkeitsrate, bei infizierten Personen ausfiel (Kormann 2020). Zudem waren die Ausbreitungswege des Virus noch weitgehend unbekannt. In dieser Situation kommt das Vorsorgeprinzip zum Tragen: lieber eine Vorsichtsmaßnahme zu viel einleiten als hinterher schmerzhaft zu erfahren, dass „man das Virus nicht ernst genug genommen hat.

Häufig bleibt bei hoher Ungewissheit nur die Möglichkeit, nicht die vermuteten Wirkungen, sondern die Eigenschaften des Risikos selbst als Kriterien für die Anwendung des Vorsorgeprinzips zu nutzen. Das könnten beispielsweise bekannte Stoffeigenschaften sein, wie Toxizität, Entflammbarkeit oder ein besonders hoher oder niedriger PH-Wert (Wissenschaftlicher Beirat der Bundesregierung „Globale Umweltveränderungen“ 1999, S. 53 ff.). Auf Corona übertragen bedeutet dies, den Virus vorrangig auf seine Ausbreitungsgeschwindigkeit und zeitliche Persistenz hin zu untersuchen, um auf dieser Basis entsprechende Präventionsmaßnahmen zu treffen (Phull 2020).

Institutionell macht es Sinn, die Frage nach der richtigen Balance zwischen zu viel und zu wenig Vorsicht bei der Bewertung der noch unsicheren Folgen nicht allein den Wissenschaftlern oder auch den Behörden zu überlassen. Denn die Frage der Verhältnismäßigkeit lässt sich nicht wissenschaftlich eindeutig beantworten, sondern setzt eine auf Werten basierende Abwägung voraus. Dazu müssen diejenigen, die von einem Risiko betroffen sind, auch mit in die Urteilsfindung einbezogen werden. Das geht natürlich nur, wenn dazu die institutionellen und prozessualen Rahmenbedingungen bereits vor Eintritt der Krise bestehen. Die deutsche Risikokommission hat in ihrem Endgutachten, das im Juni 2003 dem Umwelt- und dem Gesundheitsministerium vorgelegt wurde, zu diesem Zweck die Einrichtung eines Risikorates vorgeschlagen (Risikokommission 2003). Dieser hat die Aufgabe, eine vorsorgeorientierte Risikoabschätzung sicherzustellen und eine Umsetzung des Vorsorgeprinzips zu gewährleisten, die als rational begründbarer Kompromiss die Zustimmung der Parteien genießt, die letztlich die Kosten tragen müssen.

Die folgenden Ausführungen dienen dazu, das Vorsorgeprinzip im Rahmen der Risikoabschätzung und der Risikobewertung zu erläutern und die Umsetzung 
des Prinzips in der gegenwärtigen Coronakrise zu reflektieren. Dieser Vorschlag ist von dem Ziel getragen, die richtige Balance zwischen Leichtsinn und übertriebener Vorsicht zu finden, die für die Erhaltung der Gesundheit aber auch der Vitalität eines Landes wie Deutschland essentiell ist.

\section{Vorsorge im nationalen und internationalen Kontext}

Im deutschen Recht wird zwischen Schaden, Gefahr, Vorsorge und Restrisiko unterschieden (Rehbinder 1997). Ist ein Schaden mit einer hohen Wahrscheinlichkeit zu erwarten, spricht man von Gefahr. In diesem Falle ist Gefahrenabwehr geboten, sofern eine rechtliche Verpflichtung zur Abwendung des drohenden Schadens besteht. Ist die Wahrscheinlichkeit dagegen geringer, kann eine Regulierung des Risikos aus Vorsorgegesichtspunkten erfolgen. Bei sehr kleinen Wahrscheinlichkeiten spricht man von Restrisiken. Diese müssen - einen entsprechenden Nutzen der Aktivität vorausgesetzt - im Sinne des Allgemeinwohls hingenommen werden.

Im EU-Recht und im internationalen Recht ist das Vorsorgeprinzip im Wesentlichen auf das Problem der Ungewissheit bezogen. Obwohl es in der Literatur in vielfältiger Weise definiert und interpretiert worden ist, ist die Formulierung aus der Rio-Deklaration weiterhin die am häufigsten gebrauchte und zitierte Fassung dieses Prinzips (zitiert nach Paterson 2005):

In order to protect the environment, the precautionary approach shall be widely applied by States according to their capabilities. Where there are threats of serious or irreversible damage, lack of full scientific certainty shall not be used as a reason for postponing cost-effective measures to prevent environmental degradation (Rio Declaration 1992, Principle 15).

Ungewissheit kann eine Reihe von Ursachen haben. Bei der Bewertung von Risiken lassen sich vier Typen von Ungewissheit unterscheiden (Klinke \& Renn 2021):

- $\quad$ nicht erkannte oder nur geschätzte Variabilität bei den Endpunkten (targets) der Risikoanalysen, vor allem bei der inter-individuellen Sensibilität gegenüber gleich hohen Konzentrationen und gleichbleibender Exposition (etwa bei Exposition durch den COVID-19 Erreger);

- die Summe der systematischen und zufälligen Messfehler und der Extrapolationen bei der Interpretation von toxikologischen oder epidemiologischen Daten, bei der Dosis-Wirkungsbeziehung sowie der Berechnung der Exposi- 
tionen (statistische Konfidenzintervalle für das Infektionsrisiko zum Beispiel);

- genuin stochastische Prozesse, bei denen eine Indeterminanz vorliegt (welches Individuum infiziert wird, ist nicht klar, man kennt nur die Wahrscheinlichkeit im Rahmen einer definierten Population);

- verbleibende Unwissenheit, Ahnungslosigkeit und Setzen von Systemgrenzen der Betrachtung (verbleibende Unsicherheiten und Nicht-Wissen).

Die ersten beiden Faktoren der Ungewissheit betreffen in erster Linie die Risikoabschätzung, die beiden letzten Faktoren das Risikomanagement. In den folgenden beiden Abschnitten soll zunächst der Vorsorgegedanke für die Risikoabschätzung, dann für das Risikomanagement näher erörtert werden. Um terminologische Klarheit zu gewinnen, spreche ich im ersten Falle von vorsorgeorientierter Risikoabschätzung, im zweiten Fall von der Anwendung des Vorsorgeprinzips (bei der Bewertung und beim Management von Risiken).

\section{Vorsorge bei der Risikoabschätzung}

Wegen der Ungewissheit über die Variationsbreite in der Reaktion der vom Risiko betroffenen Endpunkte (Personen, Tiere, Pflanzen oder Biotope) sowie dem Auftreten von systematischen und zufälligen Messfehlern bedeutet vorsorgeorientierte Risikoabschätzung die Notwendigkeit zu einer konservativen, d. h. auf der sicheren Seite liegenden Beurteilung der Risiken. So werden zur Simulation der Variabilität Sicherheitsfaktoren von 2 bis zu 100.000 eingesetzt. Diese Faktoren setzen den Grenzwert um den entsprechenden Faktor niedriger als den vermuteten bzw. gemessenen Schwellenwert einer zu erwartenden negativen Wirkung. Bei der Extrapolation werden häufig konservative Annahmen, also eher vorsichtige Werte bei den Parametern, zugrunde gelegt. So werden etwa bei der Extrapolation von hohen auf niedrige Dosen häufig lineare Zusammenhänge angenommen, obwohl eine quadro-lineare oder sogar logarithmische Dosis-Wirkungs-Kurve wahrscheinlicher wäre. Gleichzeitig ist aber auch klar, dass keine probabilistische Risikoabschätzung den ungünstigsten Fall und das sensibelste Individuum einbeziehen kann. Man kann sich über die Breite des Spektrums (etwa 99\% der Individuen) streiten, aber nicht über die Notwendigkeit einer gepoolten d. h. auf Gruppen bezogenen Betrachtung (Renn 2007).

Was bedeutet das für die Vorsorge? Wenn man vom Vorsorgegedanke ausgeht, dann sollte man bei Risikoabschätzungen, soweit dies vertretbar ist, eher auf der Seite der Vorsicht irren als auf der Seite des „Wagemuts“ (Renn 2002). Dabei ist die Festlegung von sinnvollen Annahmen für vorsichtige Vorgehensweisen selbst 
nicht naturwissenschaftlich exakt vorgegeben und setzt stets ein „Werturteil“ im Sinne des Abwägens zwischen zu viel und zu wenig Vorsicht voraus. Denn maximale Vorsicht ist mit der Wahrscheinlichkeitstheorie unvereinbar; es muss stets ein vertretbares Maß an Vorsicht definiert werden, sonst müsste man im Prinzip alles, was Gefahren beinhalten kann, kategorisch verbieten. Dies kann aber niemand ernsthaft vertreten. Das vertretbare Maß an Vorsicht bei der Risikoabschätzung wird in der wissenschaftstheoretischen Literatur meist als Konvention bezeichnet (Akademie der Wissenschaften zu Berlin 1992, S. $342 \mathrm{ff}$.). Dazu gehört zunächst einmal die Definition dessen, was als Schaden bezeichnet wird und was in die Ermittlung des Risikos eingeht (adverse effect) oder die Wahl der Referenzgröße (etwa erwarteter Schaden pro Zeiteinheit oder pro gefahrenen Kilometer, oder pro Einheit Bruttosozialprodukt, usw.). Des Weiteren sind aber damit vor allem die Regeln gemeint, die Messstandorte, Messverfahren, Methoden zur Aggregation und Extrapolation von Messwerten usw. bestimmen.

Bei der Abschätzung des COVID-19 Erregers spielten am Anfang Abschätzungsunsicherheiten des Infektionsrisikos eine große Rolle (Li et al. 2020). Erst im Verlauf der Krise konnten der Grad der Gefährdung und auch die Letalität immer genauer bestimmt werden. Dagegen blieben hohe Unsicherheiten bei der Frage nach den neuen Risiken, die erst durch die Managementmaßnahmen ausgelöst werden. Denn auch der Stillstand des kulturellen, wirtschaftlichen und öffentlichen Lebens bringt Leid und Verluste mit sich. Arbeitslosigkeit, Konkurse, dramatische Einkommenseinbußen, räumliche Enge in kleinen Wohnungen und mangelnde Bewegung sind direkte Auslöser für menschliches Leid und führen zu gesundheitlichen Belastungen. Was die Risiken anbetrifft, gibt es auf beiden Seiten der Waage um die Vermeidung von Leiderfahrung. Ein kluges schrittweises Öffnen der wirtschaftlichen, sozialen und kulturellen Aktivitäten bei gleichzeitiger Wahrung der Abstandsregeln, vor allen in Innenräumen, wurde von der Bundesregierung zumindest in der ersten Welle der Pandemie als beste Umsetzung des Vorsorgegedankens angesehen, um im Saldo zwischen Infektionsschutz und Erstarrung des öffentlichen Lebens möglichst viel Leid zu vermeiden. Obwohl es sich hier um das später noch behandelte Risikomanagement handelt, spielt auch bei der Risikoabwägung der Maßnahmen die Unsicherheit über die dadurch ausgelösten Nebenfolgen eine wichtige Rolle für die später zu treffende Abwägungsentscheidung. Dafür sind eigene Konventionen und Regeln entweder vorhanden oder neu aufzustellen, um eine Kohärenz zwischen den ergreifenden Maßnahmen, also eine Stimmigkeit des gesamten Maßnahmenpakets, sicherzustellen (Migone 2020).

Aus normativer Sicht ist anzuraten, dass diese Konventionen im Rahmen des Wissenschaftssystems selbst gefunden und begründet werden sollen. Innerhalb der jeweiligen scientific communities können solche auf Vorsorge bezogenen 
Konventionen im Diskurs der betroffenen Disziplinen (einschließlich der Kulturwissenschaften) am besten ausgehandelt werden, weil hierzu entsprechender Sachverstand und Erfahrung notwendig sind. Diese Aufgabe ist im strengen Sinne keine wissenschaftliche Tätigkeit, sondern eine auf Fachkompetenz und Folgenkenntnis beruhende Wertung. Solange deutlich ist, dass solche Wertungen auch immer Wertbezüge zu außerwissenschaftlichen Referenzsystemen umfassen und diese deshalb auch reflektiert werden müssen, ist eine Festlegung von vorsorgeorientierten Konventionen durch die Wissenschaft selbst legitim und pragmatisch empfehlenswert. In Bezug auf Risikokommunikation und öffentliche Legitimation ist es dann allerdings erforderlich, auch die Plausibilität der Konventionen vor Dritten zu rechtfertigen.

\section{Vorsorge bei der Risikobewertung und beim Risikomanagement}

Die zwei letzten Komponenten der Ungewissheit, „Indeterminanz“ und „Ahnungslosigkeit“, sind für die Risikoabschätzung wenig relevant. Was ich nicht weiß, kann ich auch nicht wissenschaftlich abschätzen. An dieser Stelle setzt die internationale Diskussion um das auf Risikomanagement bezogene Vorsorgeprinzip an. Nahezu alle Definitionen des Vorsorgeprinzips betonen die Notwendigkeit von regulativen Maßnahmen (Handlungen), bevor zweifelsfrei feststeht, dass und ob ein „intolerables“ Risiko besteht (siehe Übersicht in: Gilbert 2019). Solche Maßnahmen sind umso notwendiger, je größer die Eingriffstiefe der riskanten Aktivität ist und je irreversibler die Folgen sind. Risikobegrenzung ist in diesem Falle nicht auf eine Reduzierung des Risikos bezogen, sondern vielmehr auf eine Situationsveränderung, die so weit wie möglich Irreversibilitäten vermeidet. In der internationalen Literatur wird dieser Ansatz auch gerne als Resilienzstrategie bezeichnet (Linkov et al. 2014). Mit regulativen Maßnahmen soll das System der Risikokontrolle robust und weniger verwundbar gemacht werden.

Das Gebot der Vorsorge gegen noch unbekannte Wirkungen von Risiken und Bedrohungen wie Pandemien wird in zwei unterschiedlichen Ausprägungen zur Geltung gebracht (Akademie der Wissenschaften zu Berlin 1992, S. 370 ff.):

(1) Das Prinzip der geringstmöglichen Exposition (im englischen Sprachraum als „ALARA-Prinzip“ bekannt; as low as reasonably achievable): nach diesem Grundsatz muss jede Exposition so weit wie möglich reduziert werden, wobei die Grenze der Möglichkeit bei dem gerade noch wirtschaftlich und sozial vertretbaren Aufwand zur Reduktion liegt. Im Falle von COVID-19 bedeutet dieser Grundsatz, die Gelegenheiten für eine Exposition zu minimieren, etwa 
durch besonders „strikte“ Abstandsregeln oder einem konsequent durchgehaltenen Mund- und Nasenschutz.

(2) Der Stand der Technik: Nach diesem Grundsatz muss jede verfügbare Technik genutzt werden, um das Risiko zu reduzieren. Bei Infektionskrankheiten wären dies Impfungen, Vorhalten von Isolierstationen in Krankenhäusern, eine ausreichende Menge medizinischer Schutzkleidung usw.

Die Anwendung beider Prinzipien führt bei nutzentheoretischer Sicht zu suboptimalen Lösungen, da Aufwand und Nutzen nicht systematisch miteinander verglichen werden. Theoretisch kann bei Anwendung dieses Grundsatzes ja nach verfügbaren Reduktionsoptionen ein potentiell gefährlicher Virus in großen Mengen emittiert werden (weil es keine Schutztechnik gibt) oder auch umgekehrt ein an sich wenig schädlicher Virus mit großem Aufwand begrenzt werden, nur weil es technisch und medizinisch möglich ist.

Ähnliches gilt auch für das ALARA-Prinzip: An welchem Punkt eine Reduktion nicht mehr vernünftigerweise vertretbar ist (unbestimmtes Rechtsprinzip), ergibt sich entweder aus einer formalen Analyse der systematischen Abwägung von Nutzen und Risiken (die aber bei hoher Unsicherheit nicht vorgenommen werden kann) oder als Resultat einer Betrachtung des Aufwandes zur Risikoreduktion, gleichgültig ob damit ein wirklicher Nutzen für Gesundheit und Umwelt verbunden ist.

In der neueren Diskussion sind neben den klassischen Elementen der Minimierung der Exposition und des Standes der Technik innovative Formen der Risikoregulierung nach dem Vorsorgeprinzip entwickelt worden. Darunter sind vor allem die sogenannten Containment Methoden zu nennen, nach denen Risikomaßnahmen, etwa erste Impfungen, erst langsam in kontrollierten Umgebungen eingeführt werden, bis einigermaßen klar ist, ob ein zum Zeitpunkt der Einführung nicht unplausibles Risiko (wie Nebenwirkungen der Impfung) auch in Wirklichkeit besteht oder nicht (Chen 2020). Mit der Begrenzung in Raum und Zeit werden mögliche Irreversibilitäten vermieden oder zumindest deren Auswirkungen zeitlich und räumlich begrenzt. In der Diskussion sind auch neue Haftungsund Versicherungsmodelle, die Risikoverursacher dazu motivieren sollen, mögliche Risiken frühzeitig zu erkennen und diese kontinuierlich im Sinne eines Monitorsystems zu beobachten (Blumenthal et al. 2020). Dabei können auch öffentlich Zuschüsse für die Versicherungsprämien geleistet werden, wenn damit dem Gemeinwohl im Sinne von Public Health gedient ist.

Besonders interessant ist auch die Möglichkeit, bei hoher Ungewissheit über noch nicht bekannte Wirkungen die Eigenschaften des Risikos selbst als Kriterium für die Anwendung des Vorsorgeprinzips zu nutzen. Der Wissenschaftliche Beirat Globale Umweltveränderungen hat in seinem Jahresgutachten von 1998 vorge- 
schlagen, bei hoher Unsicherheit über Wahrscheinlichkeit und Ausmaß eines Risikos die Kriterien Ubiquität, Persistenz und Irreversibilität als Ersatzvariable für die Beurteilung von Risiken heranzuziehen; und zwar in den Fällen, in denen über Wirkungen noch keine hinreichend genaue Kenntnis vorliegt (Wissenschaftlicher Beirat der Bundesregierung „Globale Umweltveränderungen“ 1999, S. $53 \mathrm{ff}$.). In einem von der EU finanzierten Projekt entwickelte ein Team um den Chemiker Ulrich Müller-Herold ein quantitativ messbares Filtersystem, das auf der Basis bestimmter Stoffeigenschaften (hazards) eine Vorauswahl nach dem Vorsorgeprinzip ermöglicht (Müller-Herold et al. 2005).

Institutionell macht es Sinn, die Frage nach der richtigen Balance zwischen zu viel und zu wenig Vorsicht bei der Bewertung der noch unsicheren Folgen nicht allein den Wissenschaftlern oder auch den Behörden zu überlassen. Da bestimmte Gruppen - bei zu viel Vorsorge sind es die Nutznießer des Risikos; bei zu wenig Vorsorge die Geschädigten des Risikos - die Folgekosten für diesen Balanceakt tragen müssen, sollten diese Gruppen auch bei der Festlegung des Ausmaßes der Vorsorge eingebunden werden (Klinke \& Renn 2014). Eine Expertengruppe der damaligen Akademie der Wissenschaften zu Berlin hatte zu diesem Zweck die Einrichtung eines Risiko- und Umweltrates (analog dem Modell des deutschen Wissenschaftsrates) vorgeschlagen (Akademie der Wissenschaften zu Berlin 1992, S. 342 ff. sowie S. 475 ff.). Die erste Instanz (im Wesentlichen Wissenschaftler) sollte die vorsorgeorientierte Risikoabschätzung sicherstellen, die zweite Instanz eine Umsetzung des Vorsorgeprinzips im Sinne einer rational begründbaren Abwägung durch die vom Nutzen und den Kosten von Regulierungen betroffenen Gruppen gewährleisten. Dieser Vorschlag wurde von der Ad-hoc-Risikokommission des Bundes, die von 2001 bis 2003 die Bundesregierung beraten hat, aufgegriffen. Dem Bund wurde empfohlen, einen Risikorat einzurichten, der diese Aufgabe übernehmen soll (Risikokommission 2003). Leider wurde diese Empfehlung im Verlauf der politischen Diskussion nicht weiter verfolgt. Dagegen haben anderen Länder wie etwa die Niederlande diesen Gedanken aufgegriffen und im Sinne eines vergleichenden „national risk assessment“ auch methodisch weiterentwickelt.

\section{Schlussbetrachtung}

Risikoabschätzung und Risikomanagement nach dem Vorsorgeprinzip sind Instrumente, um Mensch und Umwelt gegen ungewisse Bedrohungen und Risiken abzusichern. Gleichzeitig sind aber immer Abwägungen vorzunehmen, um kulturelle, wirtschaftliche und soziale Ziele nicht zu gefährden und das Prinzip der Verhältnismäßigkeit aufrecht zu erhalten. Die Gesellschaft muss bei aller Risi- 
kovorsorge von der Vorstellung Abstand nehmen, die Wissenschaft könne alle gefährlichen Ereignisse und Entwicklungen vorhersagen und durch präventives Handeln ausschließen. Risikoanalysen sind bestenfalls in der Lage, die möglichen Bedrohungen zu identifizieren und Schwachpunkte im System aufzudecken. Zudem helfen uns Risikoabschätzungen, bessere Entscheidungen im Zielkonflikt zwischen den Nebenfolgen der Risikoübernahme (etwa Exposition im Falle eine Pandemie) und den Nebenfolgen der eingeleiteten Schutzmaßnahmen (wirtschaftliche, soziale, kulturelle Folgen) zu treffen.

Das Vorsorgeprinzip kann dabei eine wichtige Rolle spielen. Bei hoher Unsicherheit bedeutet das Vorsorgedenken im Bereich der Risikoabschätzung, einer eher konservativen Abschätzung der Risiken (etwa 95\% oder 99\% Perzentil auf einer Normalverteilung) den Vorzug zu geben. Hier ist es also klug, eher von pessimistischen, aber keineswegs unrealistischen Annahmen auszugehen. Dazu werden von der jeweiligen Fachwissenschaft oder von interdisziplinär besetzten Gremien Konventionen und Regeln benötigt. Für den Bereich des Risikomanagements geht es vor allem um die sorgsame Abwägung von Maßnahmen nach dem Prinzip der Verhältnismäßigkeit, wobei bei hoher Unsicherheit Begleitmaßnahmen wie Containment, striktes Monitoring oder Expositionsvermeidung gerechtfertigt sind.

Ausreichende Kapazitäten, Diversifizierung und Flexibilisierung sind drei zentrale Mittel, um Systeme auch gegenüber immer wieder auftretenden Überraschungen anpassungsfähig und resilient zu gestalten. Dies gilt vor allem bei den vorbereitenden Planungen (preparedness) vor Eintritt der Krise. Das Gesundheitssystem muss mit besonderen Stressbelastungen umgehen können, es muss Notfallpläne geben, die im Ernstfall auch konsequent und zügig umgesetzt werden können, und es bedarf einer antizipativen Planung, die auch bei hohem Zeitdruck kohärentes politisches Handeln ermöglicht. Doch auch bei bester Vorsorge werden Gesellschaften mit einem gewissen Grad an Ungewissheit und Ambivalenz leben müssen.

Was ergibt sich aus dieser Problemsicht für die Umsetzung des Vorsorgeprinzips? Je komplexer die Auswirkungen einer Bedrohungssituation ausfallen, desto wichtiger sind die Instrumente der Vorsorge im Bereich der Risikoabschätzung. Je unsicherer die Folgen von möglichen Gegenmaßnahmen und je breiter gefächert die möglichen Nebenfolgen von risikoreduzierenden Maßnahmen, etwa im Falle eines Lockdowns, sind, desto wichtiger sind Maßnahmen eines vorsorgenden Risikomanagements. Und je umstrittener die verordneten Maßnahmen zur Risikoreduzierung sind, desto eher muss ein Diskurs mit den maßgeblichen Gestaltungskräften der Gesellschaft über die Akzeptabilität der jeweiligen Maßnahmen geführt werden. Dies sollte aber schon vor der Krise vorbereitet sein, weil langwierige Diskurse unter Zeitdruck nicht möglich sind. Interessant in 
diesem Zusammenhang ist die Tatsache, dass die Bundesregierung schon 2013 in einer Bundesdrucksache die Notwendigkeit einer umfassenden Vorsorgestrategie gegen eine Pandemie in Aussicht gestellt hat (Deutscher Bundestag 2013). Dazu ist es aber zumindest in dem notwendigen Detaillierungsgrad nie gekommen.

Ob diese frühzeitige Ausbalancierung der Vorsorgemaßnahmen gelingt, hat nicht nur Einfluss auf die weitere Entwicklung der Risikoanalyse als Mittel der Zukunftsvorsorge. Vielmehr entscheiden sich hier die Chancen und Möglichkeiten einer Gesellschaft: Inwieweit wird es ihr möglich sein, in Zeiten schnellen technischen und ökologischen Wandels in eigener Verantwortung und mit Blick auf die als wesentlich erkannten Werte des Menschseins verantwortungsvoll handeln zu können?

\section{Literatur}

Akademie der Wissenschaften zu Berlin (1992): Umweltstandards. Grundlagen, Tatsachen und Bewertungen am Beispiel des Strahlenrisikos. Berlin: De Gruyter.

Bennett, Paul G. (2000): „Applying the Precautionary Principle: A Conceptual Framework“. In: M. P. Cottam/D. W. Harvey/R. P. Pape/J. Tait (Hrsg.): Foresight and Precaution, Bd. 1. Rotterdam \& Brookfield: A. A. Balkema, S. 223-227.

Blumenthal, David/Fowler, Elizabeth J./Abrams, Melinda et al. (2020): „Covid-19 - Implications for the Health Care System“. In: New England Journal of Medicine 383(15), S. 1483-1488.

Chen, Wangxue (2020): „Promise and Challenges in the Development of COVID-19 Vaccines“. In: Human Vaccines \& Immunotherapeutics 16(11), S. 2604-2608.

Deutscher Bundestag (2013): Drucksache17/12051. Unterrichtung durch die Bundesregierung. Bericht zur Risikoanalyse im Bevölkerungsschutz 2012. Berlin: Deutscher Bundestag, https://dipbt.bundestag.de/dip21/btd/17/120/1712051.pdf, besucht am 13.1. 2020.

Elliott, E. Donald/Charnley, Gail (2002): „Risk versus Precaution: Environmental Law and Public Health Protection“. In: Environmental Law Reporter 32, S. 10363-10366.

Gilbert, Steven G. (2019): „Precautionary Principle“. In: Philip Wexler (Hrsg.): Information Resources in Toxicology. New York, NY: Academic Press, S. 489-494.

Klinke, Andreas/Renn, Ortwin (2014): „Expertise and Experience: A Deliberative System of a Functional Division of Labor for Post-normal Risk Governance“. In: Innovation: The European Journal of Social Science Research 27(4), S. 442-465.

Klinke, Andreas/Renn, Ortwin (2021): „The Coming of Age of Risk Governance“. In: Risk Analysis, 41 (3), S. 544-557

Kormann, Carolyn (2020): „From Bats to Human Lungs, the Evolution of a Coronavirus“. The New Yorker. https://www.newyorker.com/science/elements/from-bats-to-human-lungsthe-evolution-of-a-coronavirus, besucht am 3.4.2020.

Li, Qun/Guan, Xuhua/Wu, Peng et al. (2020): „Early Transmission Dynamics in Wuhan, China, of Novel Coronavirus-Infected Pneumonia“. In: New England Journal of Medicine 382(13), S. $1199-1207$.

Linkov, Igor/Bridges, Todd/Creutzig, Felix et al. (2014): „Changing the Resilience Paradigm“. In: Nature Climate Change 4(6), S. 407-409. 
Migone, Andrea Riccardo (2020): „The Influence of National Policy Characteristics on COVID-19 Containment Policies: A Comparative Analysis“. In: Policy Design and Practice 3(3), S. $259-276$.

Müller-Herold, Ulrich/Morosini, Marco/Schucht, Olivier (2005): „Choosing Chemicals for Precautionary Regulation: A Filter Series Approach“. In: Environmental Science \& Technology 39(3), S. 683-691.

Paterson, John (2005): Sustainable Development, Sustainable Decisions and the Precautionary Principle. Aberdeen: University of Aberdeen.

Phull, Amit (2020): „What We Must Learn From Wuhan“. Op-Med. https://opmed.doximity.com/ articles/what-we-must-learn-from-wuhan?_csrf_attempted=yes, besucht am 20.3.2020.

Rehbinder, Eckard (1997): „Stoffrecht“. In: Arbeitskreis für Umweltrecht (Hrsg.): Grundzüge des Umweltrechtes. Berlin: Erich Schmidt, S. 13/001-013/051.

Renn, Ortwin (2002): „Vorsorge als Prinzip: Besser in der Vorsicht irren als im Wagemut“. In: GAIA - Ökologische Perspektiven in Natur-, Geistes- und Wirtschaftswissenschaften 1, S. $44-46$.

Renn, Ortwin (2007): „Precaution and Analysis: Two Sides of the Same Coin?“. In: EMBO Reports 8(4), S. 303-305.

Risikokommission (2003): Abschlussbericht der Risikokommission „Neuordnung der Verfahren und Strukturen zur Risikobewertung und Standardsetzung im gesundheitlichen Umweltschutz der Bundesrepublik Deutschland“. München: Bundesanstalt für Strahlenschutz, https://doris.bfs.de/jspui/handle/urn:nbn:de:0221-201004071338, besucht am 13.1.2021.

Wissenschaftlicher Beirat der Bundesregierung „Globale Umweltveränderungen“ (1999): Welt im Wandel: Strategien zur Bewältigung globaler Umweltrisiken. Jahresgutachten 1998. Berlin: Springer. 